\title{
Design and implementation of image retrieval system based on block color moments and GLCM
}

\author{
Zhi-hui Wang, Xiao-li Ge, Wen-bo Xu, \\ Yue-jiao Fan, Qi-la Sa, Jin-lin Li \\ College of Electronic Information and Engineering \\ Inner Mongolia University \\ Hohhot 010021, China \\ E-mail: wzhbit2007@163.com
}

\author{
Xiao-Li Ge \\ Branch Ordos China Telecom Corporation Ltd \\ Ordos 017000, China \\ E-mail: gexiaoli1204@163.com
}

\begin{abstract}
In this paper, aiming to overcome the shortcoming of global color histogram which lack of color spatial distribution information, block color moment is used to describe the color feature. In order to extract the texture feature, Canny operator for edge detection is applied firstly, and then the gray level co-occurrence matrix of the edge image is constructed. The results prove that comprehensive of color and texture in image retrieval method overcomes the limitations of using a single, at the same time it improves the retrieval accuracy and speed.
\end{abstract}

Keywords- image retrieval; color moments; edge detection; GLCM(gray level co-occurrence matrix)

\section{INTRODUCTION}

Many times, we need quickly and accurately find one or some specific images a huge image library, this is image retrieval. What is CBIR (content-based image retrieval) ? It is named compared to TBIR (text-based image retrieval) method. TBIR searches the image not by image itself, but through the matching degree of key word and image tag. However, the image tag is manual annotated, so there is more subjective influence [1]. On the other hand, CBIR technology based on image's own visual features such as color, texture and shape, has become a hot research topic in the field of current information[2].

Color features is one of the most basic visual information contents of the underlying image[3]. All the low-level visual $\mathrm{C}$ in the image, relate to other features such as texture, shape, contour, the color are of significant and stable visual features [4]. In order to overcome the shortcomings of the global color histogram which lack of color spatial distribution information, the detail of the global color histogram is analyzed in the reference paper [5].

Certainly, many researchers have found that image retrieval can get better result using two or more features of image [6-9].

In this paper block color moment is used to describe the color feature. Image texture feature is another important lowlevel visual features. In view of the disadvantage of large amount calculate of the gray level co-occurrence matrix method, this paper puts forward a image retrieval method based on combination of gray level co-occurrence matrix and
Canny operator, which can greatly reduce the computing time and have a good retrieval result which of rich texture.

\section{COLOR FEATURE EXTRACTION}

\section{A. Color Space Selection and Transformation}

RGB color model is commonly used in image processing progress, the color information collected by the existing image acquisition device is RGB values, other color spaces used in image processing also transform from RGB color space. But the RGB color space is not straightforward, which is hard to judge the color represented by the value from the RGB values. Therefore, RGB color space is not consistent with the perception of color psychology. But HSV color space is a kind of color model of visual perception ,the detail of this color model is analyzed in the reference paper [8],which is much closer to the people's experience and perception of color than RGB. It mainly includes three elements: Hue $(\mathrm{H})$, Saturation $(\mathrm{S}), \operatorname{Value}(\mathrm{V})$. Among them, the Hue affects person's visual perception; the Saturation reflects color depth; the Value reflects the light and shade.

In Fig. 1, Hue is described by the surrounding color hexagon angle, which uses red axis as $0^{\circ}$ axis ordinary. Along with the axis of the cone to measure values. When $\mathrm{V}$ $=0$,The end of the shaft is black; When $\mathrm{V}=1$, the end of the shaft is white; Thus, the axis represents all the gray scale. Saturation refers to the distance to the Value shaft.

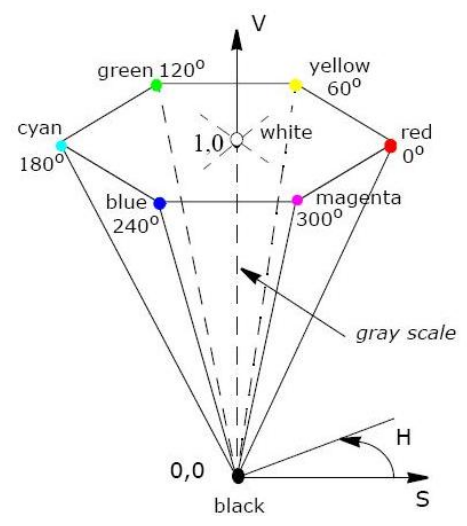

Figure 1. HSV color model 
One of transformations from RGB color space to HSV color space is proposed by references [10], the specific conversion formula is as follows:

$$
\begin{aligned}
& V=\max (R, G, B) \\
& S=\frac{V-\min (R, G, B)}{V} \\
& H^{\prime}=\left\{\begin{array}{l}
5+b^{\prime}, R=\max (R, G, B) \text { and } G=\min (R, G, B) \\
1-g^{\prime}, R=\max (R, G, B) \text { and } G \neq \min (R, G, B) \\
1+r^{\prime}, G=\max (R, G, B) \text { and } B=\min (R, G, B) \\
3-b^{\prime}, G=\max (R, G, B) \text { and } B \neq \min (R, G, B) \\
3+g^{\prime}, B=\max (R, G, B) \text { and } R=\min (R, G, B) \\
5-r^{\prime}, \text { other }
\end{array}\right. \\
& H=60 \times H^{\prime} \\
& \text { here: } r^{\prime}=\frac{V-R}{V-\min (R, G, B)}, g^{\prime}=\frac{V-G}{V-\min (R, G, B)} \text {, } \\
& b^{\prime}=\frac{V-B}{V-\min (R, G, B)} \\
& H \in[0,360], S \in[0,1], V \in[0,1] .
\end{aligned}
$$

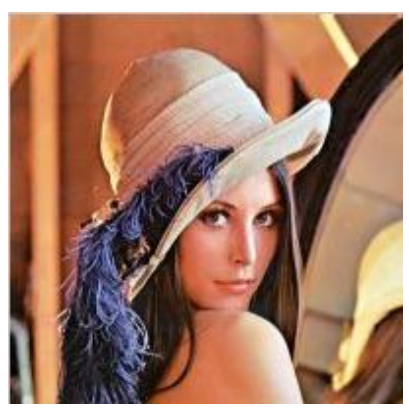

Figure 2. Original image



Figure 3. HSV image

In order to reduce the amount of calculation and the effect of Saturation and Value to retrieval result, according to human visual perception characteristics of color, this paper respectively quantifies $\mathrm{H}, \mathrm{S}, \mathrm{V}$ three components. The detail of the quantification is analyzed in the reference paper [4], In this study, The Hue is divided into 16, Saturation is divided into 4, Value is divided into 1:

$$
\mathrm{L}=16 \mathrm{H}+4 \mathrm{~S}+\mathrm{V}
$$

\section{B. Block Color Moment Algorithm}

Color moment is putted forward by Stricker and other scholars, which is an effective color feature that describes color statistical distribution features. The detail of this algorithm is analyzed in the reference paper [11].Which is the color values of pixels, color quantization is not required, so there is no loss of image color information, and the characteristic vector dimensions are much less compared with color histogram, which is convenient to calculate. Through verification, the color distribution information mainly concentrated in low moments, therefore, only use the first three moments of color can be a good color distribution of image. Among them, the first order central moments, the second order central moment and the third order central moments respectively express average color, standard deviation, three root asymmetry of image or sub-domain image. Whose expressions are:

$$
\begin{aligned}
& \mu=\frac{1}{A} \sum_{i} \sum_{j} P_{i j} \\
& \sigma=\left[\frac{1}{A} \sum_{i} \sum_{j}\left(P_{i j}-\mu\right)^{2}\right]^{1 / 2} \\
& s=\left[\frac{1}{A} \sum_{i} \sum_{j}\left(P_{i j}-\mu\right)^{3}\right]^{1 / 3}
\end{aligned}
$$

Where $A$ stands for the total number of pixels in the image, $P_{i j}$ stands for HSV pixel value that compounded by $2 \mathrm{~d}$ image space coordinates $(i, j)$.So this can build color main feature vector $(\mu, \sigma, s)$ to represent an image or the area of an image. The similarity of the images use weighted Euclidean distance D to measure, which is among corresponding color moment feature vector:

$$
D(Q, I)=w_{\mu}\left|\mu_{Q}-\mu_{I}\right|+w_{\sigma}\left|\sigma_{Q}-\sigma_{I}\right|+w_{s}\left|s_{Q}-s_{I}\right|
$$

Where $Q$ stands for query image, $I$ stands for an image of the image database, $D(Q, I)$ stands for the similarity between image $Q$ and $I, w_{\mu}, w_{\sigma}, w_{s}$ respectively stands for the weight of the mean, standard deviation, three root asymmetry characteristics.

\begin{tabular}{|l|l|l|}
\hline 1 & 2 & 3 \\
\hline 4 & 5 & 6 \\
\hline 7 & 8 & 9 \\
\hline
\end{tabular}

Figure $4 . \quad 3 * 3$ block 
the weight of the mean, standard deviation, three root asymmetry characteristics.

Although the color histogram translates invariance and simple calculates, but color histogram contains only the frequency that a particular color appear in the image, which does not show the spatial location information that a pixel located, but the color spatial distribution directly influence people's judgment about the image's similarity. In order to overcome this shortcoming, this paper adopted the $3 * 3$ block color moment, it means that according to the rectangular divide the image space to $3 * 3$ block, then for each block to extract the corresponding color features, which can build a main feature vector sequence of color distribution.

Finally, the similarity between image $Q$ and $I$, it means that weighted Euclidean distance $D_{c}$ among corresponding color moment feature vector:

$$
D_{c}(Q, I)=\sum_{i} w_{i}\left[w_{\mu}\left|\mu_{Q}-\mu_{I}\right|+w_{\sigma}\left|\sigma_{Q}-\sigma_{I}\right|+w_{s}\left|s_{Q}-s_{I}\right|\right]
$$

Where $i \in[1,9], w_{i}$ stands for the weight that the importance visual of each area.

\section{TEXTURE FEATURE EXTRACTION}

\section{A. Canny Edge Detection Operator}

In 1986,Canny operator is proposed by John Canny, the detail of this operator is analyzed in the reference paper [12],which is a kind of edge detection operator with good performance and has been applied in many fields of image processing. Canny has studied the characteristics which is required by the optimal edge detection method. The process of the edge detection is described in the literature [13]. The specific algorithm can be finished in the following four steps:

- Step1: Image filtering by Gaussian filter to filter out the noise.

- Step2: After filtering of the image, use a first order differential operator to calculate the size of the gradient $M(i, j)$ and orientation $\theta(i, j)$.

- $\quad$ Step3: The gradient uses "non-maximum suppression".

- Step4: Detect and connect the edge with double threshold algorithm.

These steps are resnectivelv shown in Fig.5, Fig.6, Fig.7 and Fig.8.

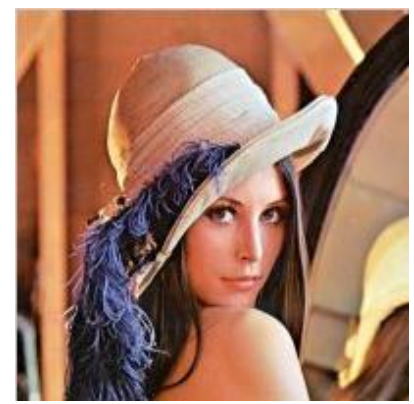

Figure 5. Original image

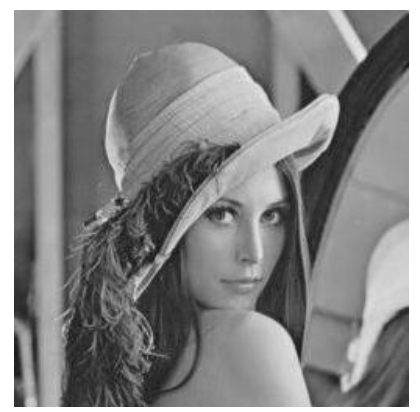

Figure 6. Processed image by Gaussian filter

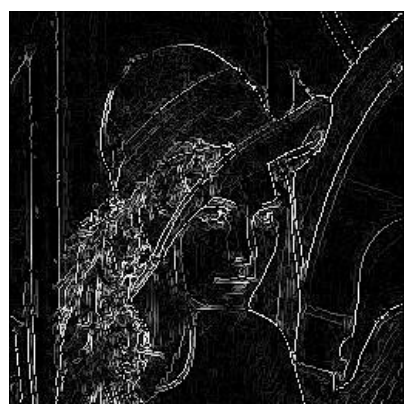

Figure 7. Processed image by "non-maximum suppression"

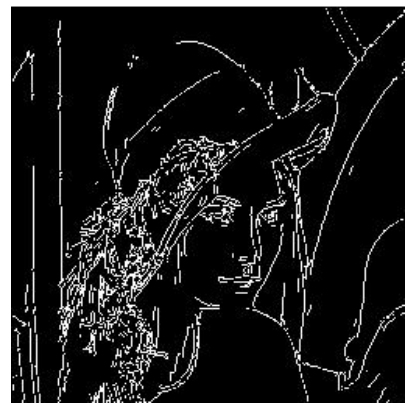

Figure 8. Processed image by Canny edge detection

\section{B. Gray Level Co-occurrence Matrix (GLCM)}

GLCM is a common way to describe texture features of images. It represents the probability that a pair of pixels respectively have the gray level $i$ and $j$ whose distance is $d$ on the direction of $\theta$ appear in the image, denoted by: $P(i, j, d, \theta)$. The process algorithm for GLCM part of this paper comes from references [14], which can reflect comprehensive information of image's gray scale about direction, adjacent interval and changing amplitude. On the basis, texture features are extracted, which are called secondary statistic.

1) Energy (ASM): 
Energy reflects uniformity and texture coarseness of the image's gray distribution.

$$
A S M=\sum_{i, j}\{P(i, j)\}^{2}
$$

\section{2) Entropy (ENT)}

Entropy is the information measure of the image, which reflects non-uniform degree or complexity of the image's texture.

$$
E N T=-\sum_{i, j}\{P(i, j)\} * \log \{P(i, j)\}
$$

\section{3) Inertia moment (CON)}

Inertia moment reflects the definition of the image and depth degree of texture's groove.

$$
C O N=\sum_{i, j}(i-j)^{2} P(i, j)
$$

\section{4) Correlation (COR)}

Correlation measures the similarity of spatial GLCM elements on the row or column direction, therefore, correlation's size reflects the local gray correlation in the image.

$$
C O R=\frac{\sum_{i, j}\left(i-\mu_{x}\right)\left(j-\mu_{y}\right) P(i, j)}{\sigma_{x} \sigma_{y}}
$$

Where $\mu_{x}, \mu_{y}, \sigma_{x}, \sigma_{y}$ are the mean and standard deviation of $P_{x}, P_{y}$ respectively, $P_{x}$ is the sum of each line's elements in the matrix $P(i, j)$, and $P_{y}$ is the sum of each column's elements in the matrix $P(i, j)$.

\section{Combining Canny Operator With GLCM}

Although GLCM is an effective method to extract the texture features, but for more gray scale images, GLCM's construction need large amount of calculation. If an image's gray scale is $\mathrm{L}$, size of $\mathrm{M}^{*} \mathrm{~N}$, in that way, the calculation of GLCM's construction is $L^{2} M N$. So in order to reduce the amount of calculation, which can properly reduce image's gray level to improve the speed of retrieval.

Canny operator, which can better keep the original image's texture features, is used to realize the original image's edge detection. This step can save the computation time of GLCM's construction. We can obtain the image's edge image after edge detection, on this basis, calculate the image's GLCM on the 4 direction of $\theta=0^{\circ}, 45^{\circ}, 90^{\circ}, 135^{\circ}$. Then the mean and standard deviation of characteristics, vectors, inertia moment, energy, entropy, correlation which is GLCM's corresponding items in the four directions are calculated, and the image's synthetical GLCM can be obtained.

$$
F=\left[\mu_{A S M}, \mu_{E N T}, \mu_{C O N}, \mu_{C O R}, \sigma_{A S M}, \sigma_{E N T}, \sigma_{C O N}, \sigma_{C O R}\right]
$$

Because when eight eigenvectors is measuring by the Euclidean distance, small components are easy to be ignored, so the feature vectors need normalization. This paper uses Gaussian normalization, the detail of this method is analyzed in the reference paper [6], then each component will have the same weight.

The similarity of the two pictures $(Q$ and $I)$, it means that Euclidean distance $D$ among GLCM feature vector:

$$
\begin{aligned}
D_{t}(Q, I)= & \left|\mu_{A S M_{Q}}-\mu_{A S M_{I}}\right|+\left|\mu_{E N T_{Q}}-\mu_{E N T_{I}}\right| \\
& +\left|\mu_{C O N_{Q}}-\mu_{C O N_{I}}\right|+\left|\mu_{C O R_{Q}}-\mu_{C O R_{I}}\right| \\
& +\left|\sigma_{A S M_{Q}}-\sigma_{A S M_{I}}\right|+\left|\sigma_{E N T_{Q}}-\sigma_{E N T_{I}}\right| \\
& +\left|\sigma_{C O N_{Q}}-\sigma_{C O N_{I}}\right|+\left|\sigma_{C O R_{Q}}-\sigma_{C O R_{I}}\right|
\end{aligned}
$$

\section{MultiPle Feature Fusion Algorithm}

Different users have different retrieval requirements for the same image, so image retrieval based on single feature has already not satisfy the demand of users, then the image retrieval based on the multiple feature is produced. This article has color and texture features in the comprehensive retrieval. Use $w_{c}, w_{t}$ respectively show the weight of similarity distance both color and texture features. Use $D_{c}, D_{t}$ respectively show the similarity distance both color and texture of the two image, then do the linear weighted sum on color and texture feature. $D$ expresses the similarity of two kinds of image features after the weighted sum:

$$
D=\frac{w_{c}}{w_{c}+w_{t}} \times D_{c}+\frac{w_{t}}{w_{c}+w_{t}} \times D_{t}
$$

From the above equation, we can know that different weight sizes of features' similarity stand for the important degree with different features of images in the retrieval process. At the time of retrieval, the background of the system should choose the appropriate weight ratio according to the actual characteristic of the image library.

\section{THE EXPERIMENTAL RESULTS AND ANALYSIS}

This experiment chooses 50 images in the Coral repository, This repository is proposed by references [16], which include five types: bus, building, Africa, beach and flower, which respectively choices ten images as the retrieval images. 
In this paper, we design three experiments:

- Only use of texture feature for retrieval;

- Only use of color features for retrieval;

- Comprehensively utilize color and texture feature for retrieval.

When comparing the retrieval performance of different methods, we use precision ratio, recall ratio and time as the evaluation standard of retrieval effect. Precision ratio is the proportion that the number of relevant images system returns in all the number of images returns in the process of a query. Recall ratio is the proportion that the number of relevant images system returns in the query result of test image in all related number of images in the library.

In view of the images with rich texture, using the method based on GLCM and the method suggested in this paper to retrieve, the result is shown in Fig.9 and Fig.10.From these pictures, we can see that the computation time of getting GLCM of an image with size of $384 * 256$ by two methods are $157.093 \mathrm{~s}$ and $2.2 \mathrm{~s}$ respectively. Thus, the proposed method in this paper can quickly calculate the texture features of images, and greatly shorten the calculation time compared with the traditional method of GLCM.

The following figures respectively shows the result of the method based on block color moments and comprehension block color moments with GLCM.

As you can see from the Fig.10, Fig.11, and Fig.12, compared with single feature retrieval, comprehensive color and texture features more fit with human's visual requirements, has a higher accuracy.

In this experiment, we change the weight of color features $W t$ and the weight of texture features $W c$ to retrieve, then counted the retrieval results to select the best weight of $W c$ and $W t$, which respectively as the right weight of the color features and texture features. Considering the average precision rate of the retrieved results in each category, this article takes the weight of $W c$ and $W t$ respectively is 0.4 and 0.6 .Then select 10 images in each category randomly to build an image library, and respectively uses the methods based on color, texture and comprehension color and texture features to retrieve, select the first nine of the most similar images as retrieval results back every time. Compare the retrieval results of three methods, the following table shows the average precision ratio of image retrieval.

We give the average precision ratio of image retrieval by different algorithms in Tab. I.

TABLE I. AVERAGE PRECISION RATIO OF IMAGE RETRIEVAL

\begin{tabular}{|c|c|c|c|c|c|}
\hline Algorithm & Bus & Building & Africa & Beach & Flower \\
\hline Color & 0.35 & 0.44 & 0.48 & 0.24 & 0.32 \\
\hline Texture & 0.58 & 0.53 & 0.72 & 0.25 & 0.51 \\
\hline $\begin{array}{c}\text { Color and } \\
\text { Texture }\end{array}$ & 0.88 & 0.59 & 0.85 & 0.45 & 0.76 \\
\hline
\end{tabular}

From Tab.1, it can be seen that comprehensive color and texture features-based retrieval is superior to single featurebased retrieval.

\section{CONCLUSIONS}

Content-based image retrieval has became a hot research in image processing field. This paper proposed a method which uses the comprehension of color and texture features to retrieve, of which, block color moments is to represent the color features, texture feature extraction is based on the Canny operator With GLCM. The results prove that compared with single feature retrieval, comprehensive color and texture features is fit with human's visual requirements, and improves the retrieval accuracy and speed.

\section{ACKNOWLEDGMENT}

This work research was financially supported by the National Natural Science Foundation of China (Grant No.61261003), and the Natural Science Foundation of Inner Mongolia Autonomous Region of China (Grant No.2015MS0610).

\section{REFERENCES}

[1] Asiyan Haimiti, Abudurexiti Haimiti, "Comparative research of image retrieval based on text and content", Journal of Capital Normal University, Natural Science Edition, vol. 33, pp. 6-9, 2012.

[2] Yan Zhou, Zeng Zeng, and Huimin Zhao, "An image retrieval method based on fine sparse adaptive matching pursuit algorithm", Electronic journal, vol. 42, pp. 2457-2466, 2014.

[3] Fanjie Meng and Baolong Guo, "Research on Content-Based Image Retrieval Technology", APPLICATION RESEARCH OF COMPUTERS, vol. 21, pp. 21-24,27, 2014.

[4] Lanchi Jang, Guoqiang Shen, and Guoxuan Shen, "Image retrieval algorithm of color histogram based on HSV blocking", Mechanical \& Electrical Engineering Magazine, vol. 26, 2009.

[5] SWAIN M J and BALLARD D H, "Color indexing", International Journal of Computer Vision, vol. 7, pp. 11-32, 1991.

[6] Weijun Dong, Mingquan Zhou, and Guohua Geng, "Image retrieval technique based on combined features", Compute Applications and Software, vol. 22, pp. 34-36, 2005.

[7] Yan Zhou, "Research on image retrieval based on color and shape features", Henan Polytechnic University, 2010.

[8] Shaoting Zhang, Ming Yang, Timothee Cour, Kai Yu, and Dimitris N. "Query Specific Fusion for Image Retrieval", Metaxas, US. vol.37, pp. 660-673, 2014.

[9] Lining Zhang, Lipo Wang, and Weisi Lin, "Geometric Optimum Experimental Design for collaborative image retrieval", Circuits \& Systems for Video Technology IEEE Transactions on, vol. 24, pp. 346-359, 2014.

[10] $\mathrm{Xu} \mathrm{Xu}$, "The image retrieval system research based on visual features", Zhejiang University Institute Of Computer, 1999.

[11] Stricker M and Orengo M, "Similarity of color images", Proc .SPIE Storage and Retrieval for Image and Video Databases, vol. 24, pp. 381-392, 1995.

[12] Jie Chen, Zhenhua Wang, and Lihua Dou, "The edge detection method of a scale adaptive", Optoelectronic Component., vol. 35, pp. 79-84, 2008.

[13] CANNY J, "A Computational Approach to Edge Detection", IEEE Trans on PAMI, vol. 8, pp. 70-76, 1986.

[14] Jingwang Jia and Zhi Zhong, "The research of image retrieval system based on GLCM", Microcomputer Information, vol.10-2, pp. 202-203, 2010 . 


\section{ATRANIIS PRESS}

15] Shujuan Zhang and Qingmin Wang, "The image retrieval research based on the characteristics", Computer And Modern, vol.1, 2010

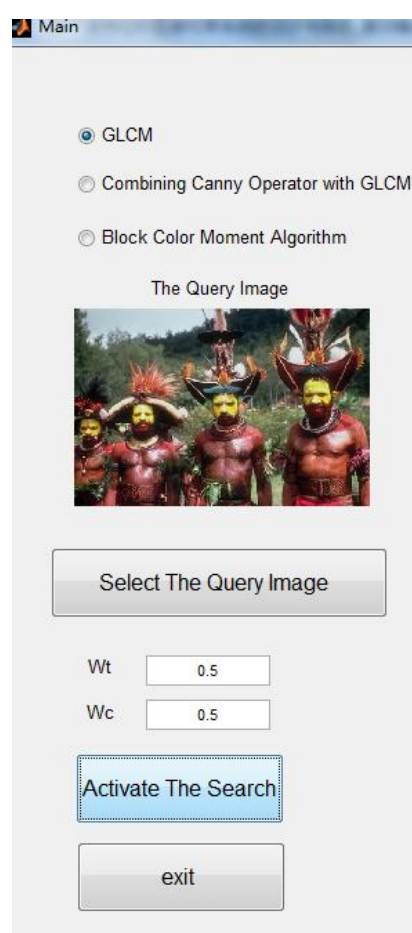

Retrieval Time 157.093 S

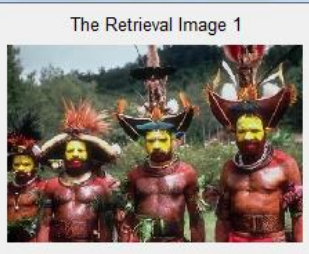

Similarity

The Retrieval Image 4



Similarity $\quad 0.95112$

The Retrieval Image 7

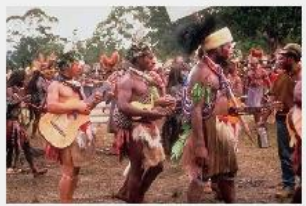

Similarity
[16] WANG J Z, "Text database in simplicity paper [DB/OL]". http://wang.ist.psu.edu/docs/related. 2011, 7.1.

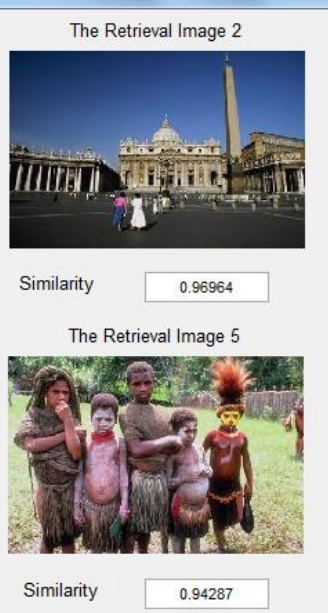

The Retrieval Image 8

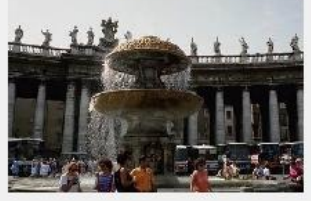

Similarity

0.83766

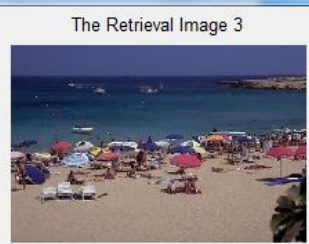

Similarity $\quad 0.95581$

The Retrieval Image 6

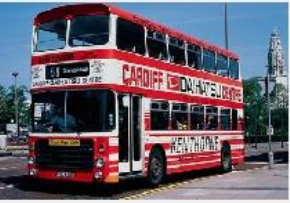

\begin{tabular}{l|l} 
Similarity $\quad 0.90857$ \\
\hline
\end{tabular}

The Retrieval Image 9



\begin{tabular}{l|l|} 
Similarity & 0.83338 \\
\hline
\end{tabular}

Figure 9. Image Retrieval Based On GLCM

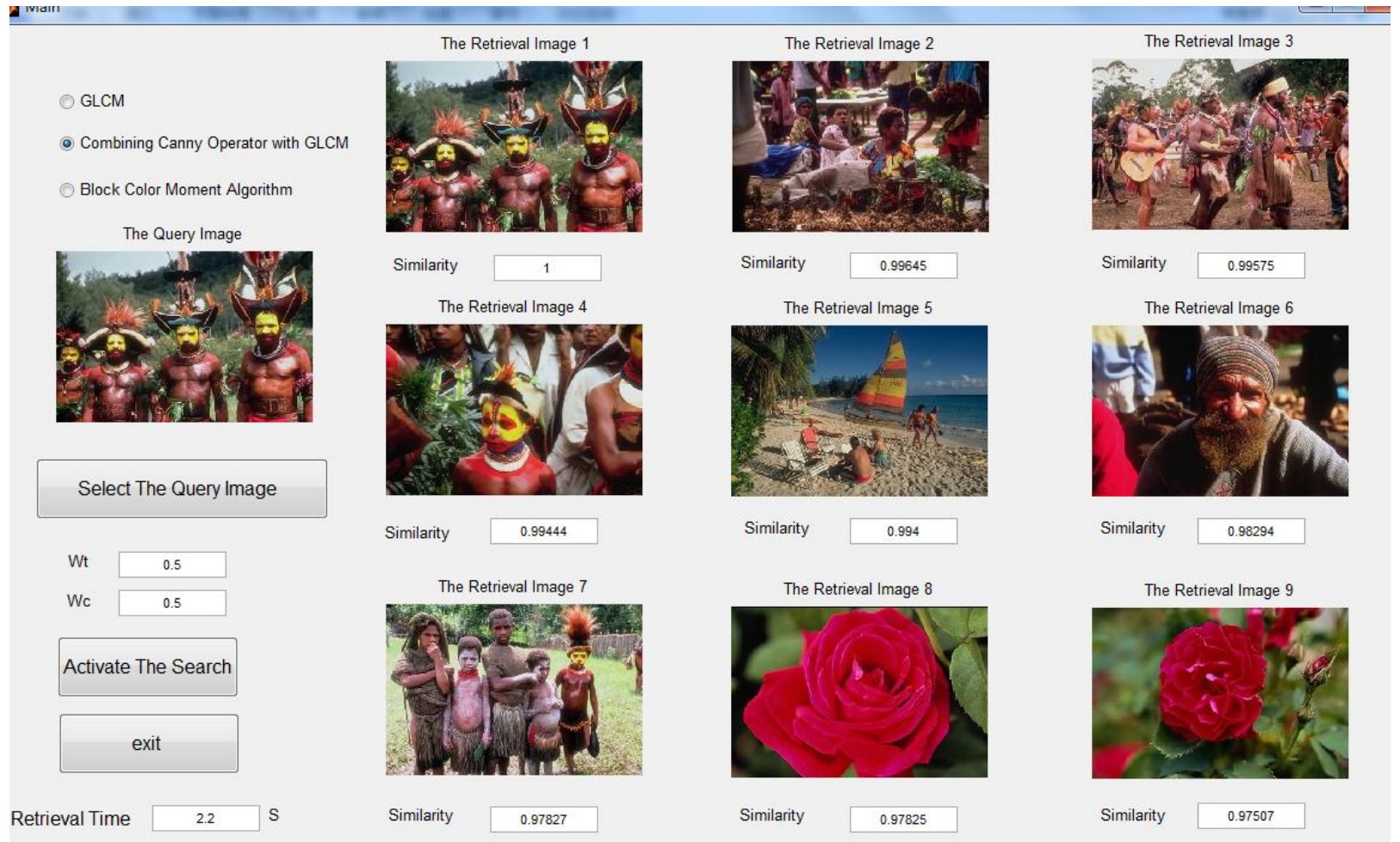

Figure 10. Image Retrieval Based On Canny Operator With GLCM 


\section{$A^{\text {AlthNIIS }}$ \\ PRESS}

Advances in Computer Science Research, (ACSR), volume 52

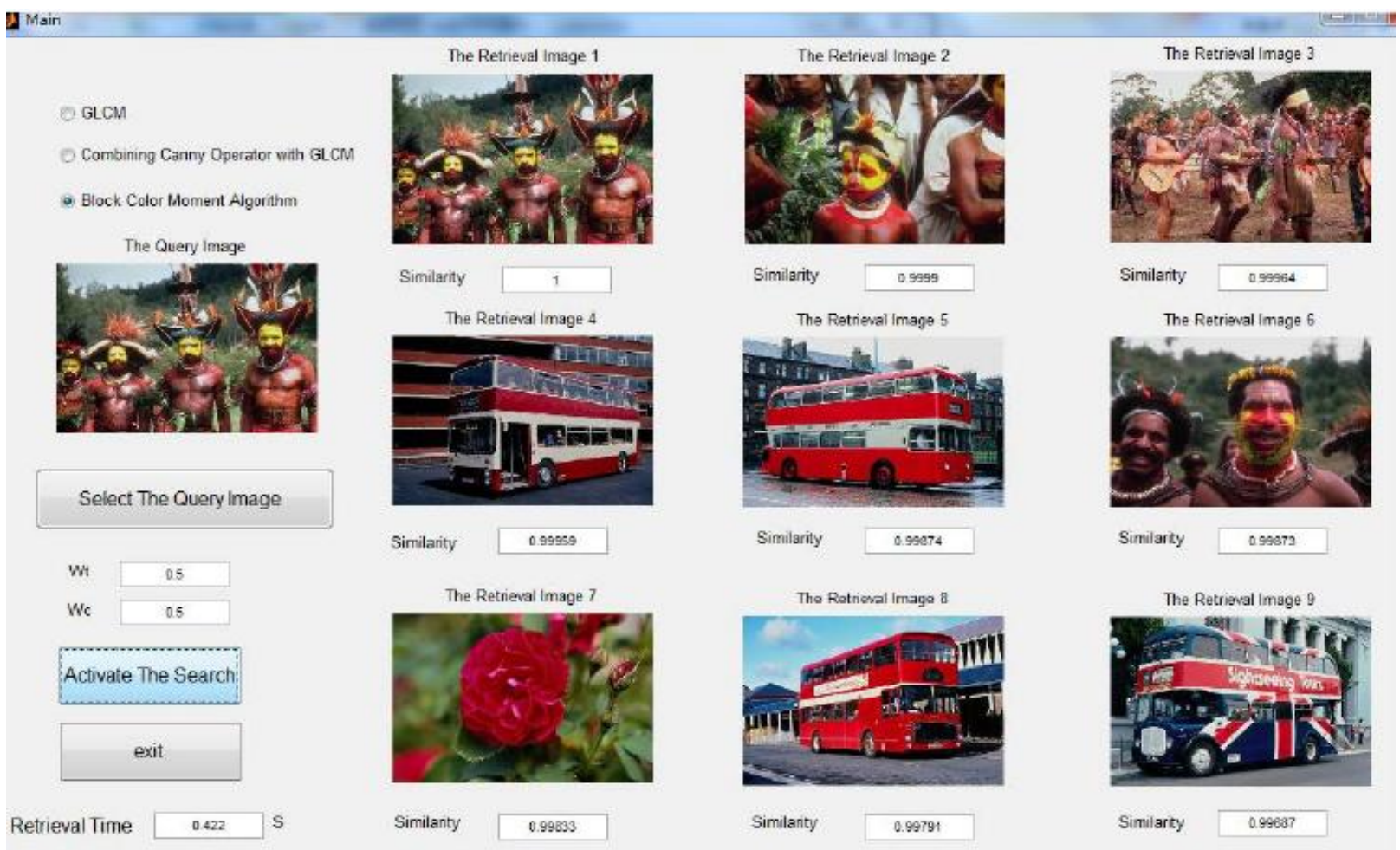

Figure 11. The Image Retrieval Based On Block Color Moments

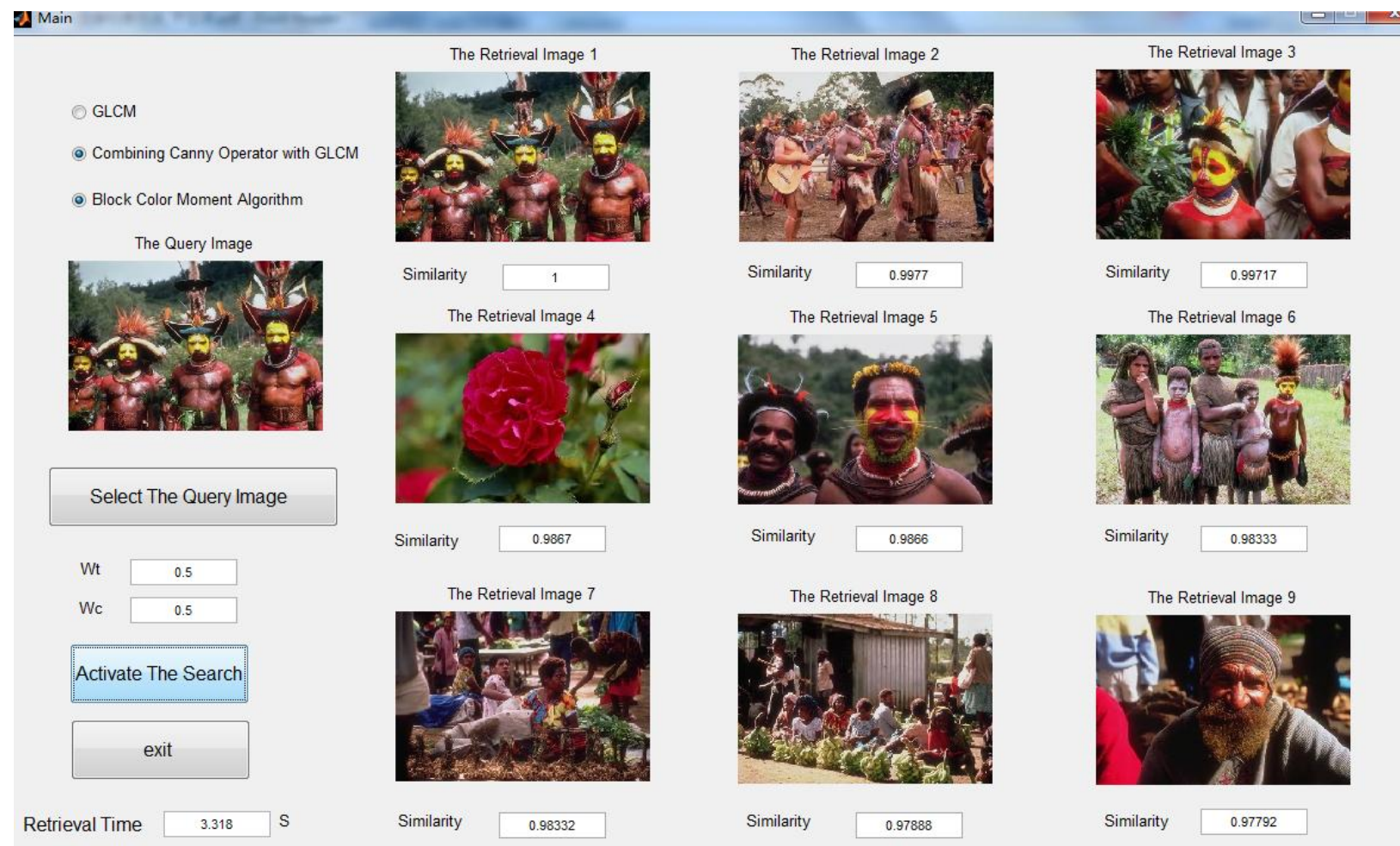

Figure 12. Image Retrieval Based On Block Color Moments and Canny Operator With GLCM 